\title{
A VARIANT MUSCLE OF THE FLEXOR COMPARTMENT OF FOREARM: A CASE REPORT
}

Yogitha Ravindranath ${ }^{1}$, Leelavathy $\mathrm{N}^{2}$

\section{HOW TO CITE THIS ARTICLE:}

Yogitha Ravindranath, Leelavathy N. "A variant muscle of the flexor compartment of forearm: a case report". Journal of Evolution of Medical and Dental Sciences 2013; Vol2, Issue 30, July 29; Page: 5700-5702.

\begin{abstract}
Variations of the muscles are well known and documented. Here we report a unilateral variation of flexor digitorum profundus in the ventral aspect of the distal forearm of an elderly male cadaver. The variant muscle presented the following attachments: the proximal end of the muscle belly was attached to the medial surface of radius beneath the flexor pollicis longus and the interosseous membrane, distal attachment was traced onto the tendon of flexor digitorum profundus. The muscle fibres were directed downwards and medially, lying superficial to the anterior interosseous nerve, vessels and superficial to the pronator quadratus. The muscle belly was innervated by a small twig of anterior interosseous nerve. None of the other forearm muscle showed variations. A profound knowledge of the anatomical variations is essential for differential diagnosis of compression syndromes as it is closely related or plastered to the anterior interosseous nerve and vessels.
\end{abstract}

KEY WORDS: variation, Flexor compartment, forearm, flexor digitorum profundus, compression syndrome.

INTRODUCTION: Muscular variants in the flexor compartment of forearm are common. These muscles are incidental in findings and most often they are detected following routine anatomical dissections or during operations. A thorough knowledge of these variations is of immense clinical importance for understanding of unusual symptoms and signs. They have been recognized as one of the possible causes of neurovascular compression syndromes or tumour like mass mimicking ganglion of the upper limb. Present study reports a variant muscle in the distal third of flexor compartment of the forearm.

CASE REPORT: During routine dissection of the upper limb of an elderly male cadaver, a variant muscle was seen unilaterally on the ventral aspect of the distal third of the flexor compartment of right forearm in a plane deep to the flexor digitorum superficialis between the flexor pollicis longus and the flexor digitorum profundus. The muscle was identified as one of the variant muscle of the flexor digitorum profundus. The muscle was carefully dissected, cleaned from end to end; the nerve supply and the relation of the surrounding structures were noted.

The variant muscle was strap like (slender) shaped and presented the following attachments: proximal end of the muscle belly was attached to the medial surface of radius beneath the flexor pollicis longus and the interosseous membrane, distal attachment was traced onto the tendon of flexor digitorum profundus. The muscle fibres were directed downwards and medially, lying superficial to the anterior interosseous nerve, vessels and superficial to the pronator quadratus (figure 1). The muscle belly was innervated by a small twig of anterior interosseous nerve. None of the other forearm muscle showed variations. 
DISCUSSION: Muscular variations in the superficial, intermediate and deep group of forearm flexors are commonly reported. The reported incidence of variant muscle was high in Blacks (89.3\%) and low in European Caucasian (33.3\%). The reported variants are, two accessory muscles inserting either into the flexor pollicis longus or flexor digitorum profundus. The reported variations arose either from the under surface of the flexor digitorum superficialis alone or with combination of fibres arising from medial epicondyle and or the coronoid process, medial epicondyle alone. These variants are innervated either by anterior interosseous nerve or directly from the median nerve $(1,2$, 3). An unusual bilateral distal and complete additional flexor profundus muscle to the index finger of the distal forearm has been described. The muscle was innervated from the median nerve (4). Present study shows considerable variation from the already reported cases of variants of flexor group of muscles. The described variant muscle could have aroused as a single muscle from the muscle Primordium (5) or as a result of persistence of the differentiated myoblast between the muscles of the forearm ${ }^{(4)}$ or due to the persistence of some cells between the flexor digitorum profundus (6).

The variant muscles of the flexor group have been reported in primates and other mammals including pigs and foxes, as a muscle that connects the medial epicondyle origin of flexor digitorum superficialis with the deep flexor group of muscles. These are formed by the incomplete cleavage of the deep layer of flexor group of muscles during embryogenesis thus representing both phylogenetically and ontogenetically the atavistic character.

CONCLUSION: To the best of our knowledge, present study reports an unusually separate muscle from the distal right forearm. The knowledge of such variations is of importance to the operating surgeons and radiologist for differential diagnosis Presence of variant muscles must be considered as a differential diagnosis for undiagnosed compression syndromes. Thus it is essential to confirm such variants pre operatively by newer imaging techniques for better management.

\section{REFERENCES:}

1. Bergman RA, Afifi AK, Miyauchi R . Muscular system. In: Illustrate Encyclopedia of Human anatomic Variation. Text, atlas and word literature, Urban \& Schwarzenberg, Baltimore, 1988.

2. Hollinshed HW. Anatomy for Surgeons. 2nd ed., Philadelphia, Harper and Row. 1969; 408-409.

3. Williams PL, Bannister LM, Berry MM, Collins P, Duson M, Dussek Je. Gray's Anatomy. 38 ${ }^{\text {th }}$ ed., Edinburgh, Churchill Livingstone.1995; 847-848.

4. Kopuz C, Gulman B, Baris S. An unusually distal and complete additional flexor profundus muscle to the index finger. Journal of anatomy 1997; 191: 465-467.

5. Sadler TW. Langman's Medical Embryology. $5^{\text {th }}$ ed., Baltimore, Williams and Wilkins. 1985; 148-151.

6. Grim M. Ultrastructure of the ulnar portion of the contrahent muscle layer in the embryonic human hand. Folia Morphologica. 1972; 113-115. 


\section{Figure: 1}

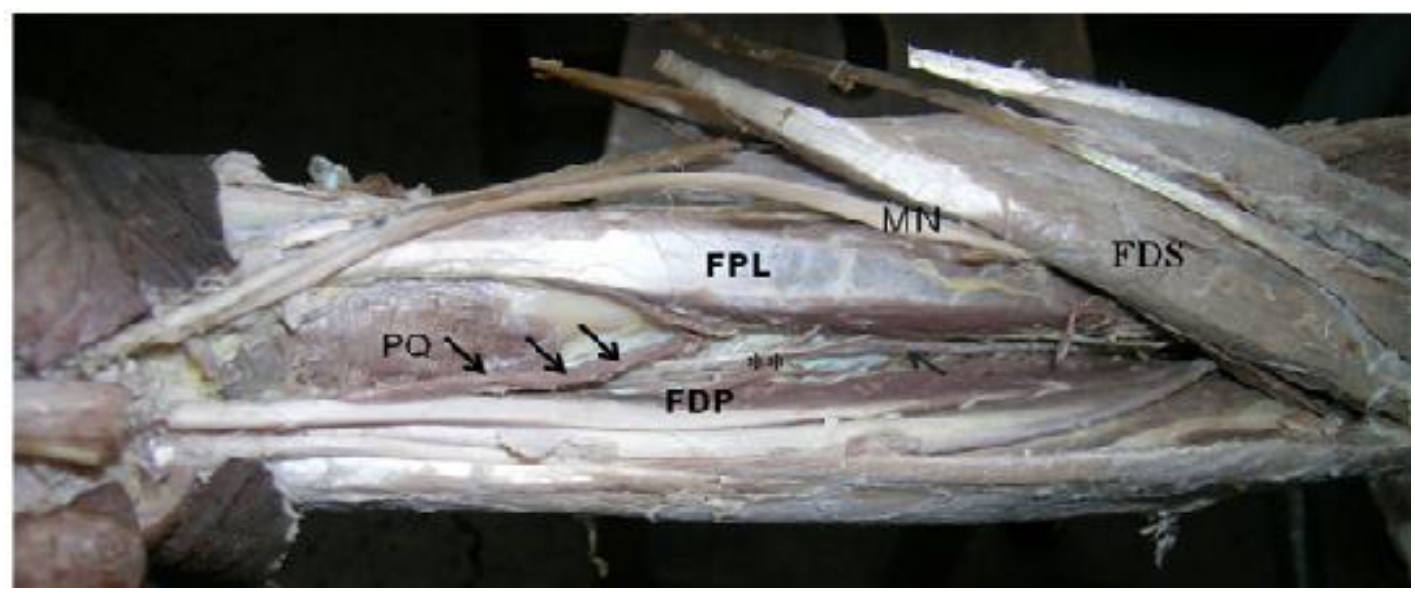

Figure 1: Arrowheads in series indicate the accessory belly of flexor digitorum profundus crossing the anterior interosseous nerve and vessels.

Asterix ${ }^{* *}$ ): anterior interosseous nerve, single arrowhead: anterior interosseous artery, FDP: flexor digitorum profundus, FPL: flexor pollicis longus, PQ: pronator quadratus, MN: median nerve.

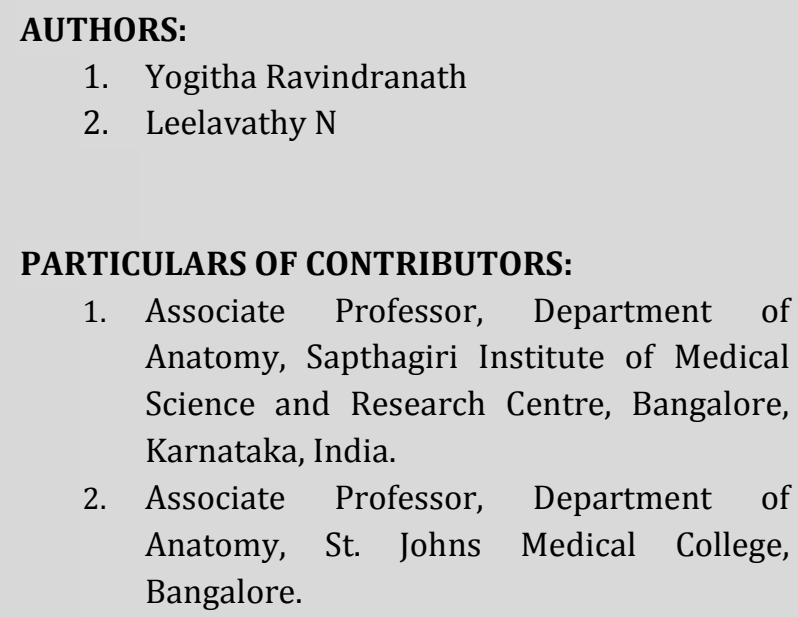

1. Yogitha Ravindranath

2. Leelavathy $\mathrm{N}$

\section{PARTICULARS OF CONTRIBUTORS:}

1. Associate Professor, Department of Anatomy, Sapthagiri Institute of Medical Science and Research Centre, Bangalore, Karnataka, India.

2. Associate Professor, Department of Anatomy, St. Johns Medical College, Bangalore.

\section{NAME ADRRESS EMAIL ID OF THE CORRESPONDING AUTHOR: \\ Dr. Yogitha R, \\ Dept. of Anatomy, St. Johns Medical College, Bangalore - 560034. \\ Email - yogi3110@gmail.com}

Date of Submission: 23/07/2013. Date of Peer Review: 24/07/2013. Date of Acceptance: 25/07/2013. Date of Publishing: 29/07/2013 